Langzeitfolgen solcher Injektionen. Die vorliegende doppelt-blinde, prospektiv randomisierte und placebokontrollierte Studie untersucht das Knorpelvolumen und den Schmerz nach intraartikulären Triamcinolon- im Vergleich zu Placeboinjektionen in das Kniegelenk.

Verglichen wurde eine Triamcinolon-Gruppe [Triam], die 3-monatlich 40 mg Triamcinolon $(40 \mathrm{mg} / \mathrm{ml})$ über 2 Jahre intraartikulär injiziert bekam, mit einer Kontrollgruppe [Placebo], die über den gleichen Zeitraum und im gleichen zeitlichen Abstand $1 \mathrm{ml}$ 0,9\%-Natriumchlorid-Lösung intraartikulär verabreicht bekam. In jede der beiden Gruppen wurden 70 Patienten eingeschlossen. Die Nachuntersuchungsdauer betrug 2 Jahre.

Einschlusskriterium war das Vorliegen einer Gonarthrose (Röntgen mit KellgrenLawrence-Kriterien Grad 2 oder 3). Ausgeschlossen wurden Patienten mit systemischen Gelenkerkrankungen, Osteonekrosen oder aktuellem Gebrauch von oralen Glukokortikoiden, Doxycyclin, Indomethacin, Glucosamin oder Chondrotoin. Ebenfalls wurden Patienten ausgeschlossen, die innerhalb der letzten 3 Monate intraartikuläre Kortison- oder Hyaluronsäureinjektionen erhalten haben. Weitere Ausschlusskriterien waren schwerwiegende Grunderkrankungen.

\section{Intraartikuläre Gaben von Triamcinolon vermindern das Knorpelvolumen}

McAlindon TE et al. Effect of intra-articular triamcinolone vs. saline on knee cartilage volume and pain in patients with knee osteoarthritis: a randomized clinical trial. JAMA 2017; 317: 19671975

Intraartikuläre Kortisoninjektionen gehören aufgrund ihres positiven Kurzzeiteffekts zu einer der Standardtherapien bei gonarthrotischen Beschwerden. Es herrscht Uneinigkeit über die
Die primären Zielparameter waren der Knorpelstatus sowie die Schmerzen im WOMAC. Die Kernspintomografie wurde jährlich durchgeführt. Der WOMAC wurde bei den alle 3 Monate durchzuführenden Kontrollen erfasst. Die Daten wurden auf den Kellgren-Lawrence-Grad und das Geschlecht bereinigt und mittels Intentionto-treat-Analyse ausgewertet. Als signifikant gewertet wurden $\mathrm{p}$-Werte $<0,5$.

Es konnte kernspintomografisch gezeigt werden, dass die Triam-Gruppe im Untersuchungszeitraum einrn signifikant größeren Verlust (in $\mathrm{mm}$ ) sowie Schaden am Knorpel im Vergleich zur Placebogruppe hatte. Gleichzeitig zeigten sich keine signifikanten Unterschiede für die klinischen Parameter (Schmerz, Funktion und Steifheit). 
Die Triam-Gruppe hatte 5 therapieassoziierte Nebenwirkungen verglichen mit 3 therapieassoziierten Nebenwirkungen in der Placebogruppe.

FAZIT

Wiederholte intraartikuläre Triamcinolon-Gaben zur Reduktion von Inflammation führen nach der vorliegenden Studie zur vermehrten Arthrosebildung.

Der kernspintomografische Knorpelverlust deckt sich mit dem in vivo und in klinischen Studien nachgewiesenen katabolen Effekt von Glukokortikoiden.

Die Autoren wiesen insbesondere auf die Einschränkungen hin, dass lediglich alle 3 Monate Daten zum Schmerzniveau erhoben wurden. Mögliche Zwischeneffekte konnten nicht detektiert werden. Zusammenfassend kann man nach den Ergebnissen der vorliegenden Studie die wiederholte Therapie mit intraartikulären Triamcinolon-Injektionen für Patienten mit symptomatischer Gonarthrose nicht empfehlen.

Dr. Hauke Horstmann, Hannover 